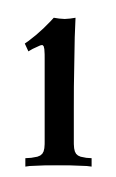

\title{
TREINTA AÑOS DE EVALUACIÓN DE CENTROS EDUCATIVOS EN ESPAÑA
}

\author{
(THIRTY YEARS OF SCHOOL EVALUATION IN SPAIN)
}

Alejandro Tiana-Ferrer

Universidad Nacional de Educación a Distancia-UNED

DOI: $10.5944 / e d u c X X 1.21419$

Cómo referenciar este artículo/How to reference this article:

Tiana-Ferrer, A. (2018). Treinta años de evaluación de centros educativos en España. Educación XX1, 21(2), 17-36, doi: 10.5944/educXX1.21419

Tiana-Ferrer, A. (2018). Treinta años de evaluación de centros educativos en España. [Thirty years of school evaluation in Spain]. Educación XX1, 21(2), 17-36, doi: 10.5944/educXX1.21419

\section{RESUMEN}

En los años ochenta del siglo xx comenzó a manifestarse un interés político por la calidad de la educación, que renovó el discurso pedagógico y llevó aparejado un refuerzo de las actividades de evaluación, al ser esta entendida como un instrumento clave para producir la anhelada mejora. Fue en ese contexto en el que comenzó a construirse en España una política común de evaluación de los centros educativos, considerados el lugar donde se encarna la tarea educativa y se debe producir la mejora. Dicha política fue cambiando a lo largo del tiempo, en función de los cambios registrados en la orientación política general, el reparto de competencias en materia de educación y las iniciativas concretas de los diversos agentes y administraciones. En este artículo se analizan las características de las políticas desarrolladas en los últimos treinta años para evaluar los centros docentes, así como de las prácticas en que se han traducido. El enfoque adoptado se inscribe en la política de la educación, centrándose en los planteamientos adoptados y las prácticas desarrolladas por las diversas administraciones. Para ello se ha recurrido a la identificación y consulta de obras académicas y de investigación, informes oficiales, normas legales y «literatura gris». Tras llevar a cabo dicho análisis, el trabajo concluye que la política adoptada a lo 
largo de estos treinta años se ha caracterizado por los avances y retrocesos, los cambios de orientación, la falta de coherencia entre las intenciones y la práctica y la insuficiencia de las acciones desarrolladas.

\section{PALABRAS CLAVE}

Política de la educación; sistema educativo español; evaluación de centros educativos; autoevaluación de centros educativos; pruebas de rendimiento; indicadores de centros educativos.

\section{ABSTRACT}

From the 1980s onwards, a new political interest started to emerge in relation to the quality of education, which renewed the pedagogical discourse and brought a reinforcement of evaluation activities, being evaluation considered a key instrument for achieving the expected improvement. In this context, a common policy on school evaluation started to be built in Spain, as schools were considered the places in which education must be incarnated and improvement achieved. This policy was changing along the years, in accordance with changes in general political orientation, distribution of responsibilities in education and concrete initiatives developed by different agents and administrations. In this article the characteristics of policies and practices developed in Spain for school evaluation in the last thirty years are analyzed. A political perspective is adopted for carrying such an analysis, focusing on the approaches taken and the practices developed by different administrations. Academic and research literature, official reports, legal regulations and "grey literature» have been identified and checked. After carrying such an analysis, the article concludes that the policy adopted can be characterized by advances and retreats, changes of orientation, lack of coherence between intentions and practices, and inadequacy of actions developed.

\section{KEY WORDS}

Educational policy; Spanish education system; school evaluation; school self-evaluation; achievement tests; school indicators. 


\section{INTRODUCCIÓN}

En la década de los ochenta del siglo xx la política de la educación comenzó a interesarse internacionalmente por la cuestión de la calidad. Numerosos factores, tanto externos como internos al sistema educativo, confluyeron para propiciar ese giro, que marcó el inicio de una época que se extiende hasta nuestros días y ha modificado profundamente el discurso pedagógico tradicional. Como consecuencia, académicos, administradores y políticos de la educación dirigieron su atención hacia los problemas ligados con la calidad y su mejora (De la Orden, 1988; Marchesi \& Martín, 1998; Organisation for Economic Co-operation and Development [OECD], 1994; Santos Guerra, 2003; Varios autores, 1994). Quizás la demostración más patente del giro que se había producido al llegar al final de esa década se encuentra en la celebración en París, en noviembre de 1990, de una reunión de los ministros de Educación de los países miembros de la Organización para la Cooperación y el Desarrollo Económico con el título High-Quality Education and Training for All (OECD, 1992), dedicada a la promoción de las políticas en favor de la mejora de la calidad de la educación obligatoria.

El desplazamiento progresivo de la atención académica y del interés político hacia la mejora de la calidad se tradujo en un refuerzo de las actividades de evaluación de la educación, al ser considerada uno de los factores más influyentes para conseguir dicha mejora. Es así como la evaluación, como nos recuerda Ernst House analizando su experiencia personal, pasó de considerarse una actividad académica de alcance limitado a convertirse en una verdadera empresa, con sus propias revistas, premios, reuniones, organizaciones y estándares (House, 1993). Puede decirse que fue esa la época de sistematización de la evaluación educativa, que marcaría profundamente su desarrollo posterior.

Entre los ámbitos atendidos por la evaluación de la educación, fue en esos años ochenta cuando se despertó el interés por evaluar los centros educativos. Con anterioridad se habían ido formalizando otros campos, como la evaluación del currículo, de los programas de enseñanza o del rendimiento de los estudiantes. Pero la conciencia del papel decisivo que desempeñan los centros y el profesorado en la mejora de la calidad llevó a situarlos en el foco de la actividad evaluadora. Es así como en países muy diversos surgieron planes, programas e iniciativas para evaluar a los centros. Como afirmaba una interesante publicación de la OECD que pasaba revista a la experiencia de siete países, España entre ellos, los centros educativos comenzaron por entonces a someterse al escrutinio público (OECD, 1995).

De ese modo, ya en los años ochenta aparecieron en España los primeros planteamientos y las primeras iniciativas de evaluación de centros educativos, 
algunos de ellos inspirados en la experiencia de otros países cercanos. Con posterioridad, esos proyectos pioneros fueron dando paso a planes más articulados y con mayores pretensiones, a cargo de diferentes administraciones y contando con la participación de distintos actores. La evaluación de centros, que se insertó por entonces en el horizonte pedagógico español, continúa llevándose a cabo en la actualidad, aunque el trayecto recorrido haya adolecido de discontinuidad, como podrá verse con mayor detenimiento.

En este trabajo se analiza la experiencia desarrollada en España en materia de evaluación de los centros educativos a lo largo de estos últimos treinta años. El enfoque adoptado se inserta en el campo de la política de la educación, esto es, no se pone el énfasis en las iniciativas singulares ni en sus características pedagógicas, contenidos y desarrollo, salvo con propósitos de ejemplificación, sino en las políticas adoptadas y los planes desarrollados por los diferentes agentes y administraciones educativas. En consecuencia, tampoco se ha pretendido dibujar un cuadro exhaustivo de las iniciativas emprendidas, aunque un buen número de ellas sean ciertamente valiosas e interesantes, quedando dicha tarea pendiente para otra ocasión. Obviamente, se atiende a los modelos propuestos en diferentes contextos y a las prácticas evaluadoras aplicadas, pero analizando primordialmente su concepción y su sentido, sus propósitos y objetivos, su inserción en el conjunto de las políticas educativas adoptadas y su impacto sobre el sistema educativo. Para abordar dicho análisis, el trabajo ha requerido la búsqueda sistemática de información acerca de los modelos propuestos y los principales planes y programas desarrollados o, al menos, de los más representativos. Las fuentes utilizadas no se han reducido a obras académicas, normativa estatal y autonómica, o documentos e informes oficiales, sino que ha sido necesario bucear en la denominada «literatura gris» e incluso recabar testimonios de actores destacados en estos procesos evaluadores.

\section{LA CONSTRUCCIÓN DE UNA POLÍTICA COMÚN DE EVALUACIÓN DE CENTROS A COMIENZOS DE LOS NOVENTA}

La primera referencia a la evaluación de los centros educativos en un documento de naturaleza política apareció en el Libro Blanco que precedió a la elaboración de la Ley General de Educación (LGE) de 1970 (Ministerio de Educación y Ciencia [MEC], 1969). Si bien allí se centraba la atención en la evaluación del rendimiento educativo, como cabía esperar de su época, se afirmaba que se debía referir tanto a la acción de los centros docentes como a la de los estudiantes. Mientras que la evaluación del alumnado correspondería a los propios centros, la de estos se atribuía a la inspección educativa, planteamiento que encontraría un profundo arraigo, como veremos. Aunque ni el Libro Blanco ni la LGE se extendían más en este 
asunto, quedaba así trazado un camino por el que se continuaría avanzando tiempo después.

Habría que esperar casi veinte años para que un nuevo documento político relevante, en este caso el Proyecto para la reforma de la enseñanza, presentado en 1987 por el Ministerio de Educación y Ciencia, volviese a abordar la evaluación de los centros docentes, aunque sin introducir grandes novedades (MEC, 1987). En el capítulo dedicado a la evaluación del proceso educativo, considerada un aspecto clave de la práctica escolar, se refería a la evaluación de los procesos de enseñanza que tienen lugar en las escuelas, añadiendo que debería abordarse desde una doble perspectiva, interna y externa. Nuevamente se asignaría a la inspección la responsabilidad de esta última, atribuyéndole una doble función, tanto evaluadora como orientadora, en la que se iría insistiendo en documentos posteriores.

Pero solo dos años más tarde, en 1989, el nuevo Libro Blanco elaborado para la preparación de la Ley Orgánica de Ordenación General del Sistema Educativo (LOGSE) de 1990 presentaba unos principios mucho más avanzados para llevar a cabo la evaluación de los centros educativos (MEC, 1989). Conviene resaltar que el documento suponía un avance sustancial en relación con los anteriores, sentando las bases para la construcción de una política de evaluación general del sistema educativo, entendida como un conjunto diversificado de actividades evaluadoras en distintos campos complementarios. En relación con la evaluación de los centros educativos, subrayaba su carácter integrado, planteando que debería sobrepasar el análisis de los resultados de los estudiantes para adentrarse en la consideración de los contextos, recursos, objetivos, estructuras y procesos, lo que permitiría adaptar la actividad evaluadora a las circunstancias concretas de cada centro. Así mismo, planteaba combinar la rendición de cuentas con la mejora de la calidad, entendidas como dos tareas indisociables. Además, insistía en el valor de la autoevaluación escolar, en tanto que mecanismo de autorregulación y mejora, que debería combinarse con la evaluación externa. Era la primera vez que se planteaba abiertamente este principio, que encontraría posteriormente un mayor desarrollo. La evaluación externa se asignaba nuevamente a la inspección técnica de educación, quien debería realizar un análisis cualitativo cuidadoso de las condiciones de los centros educativos, planteando así la función evaluadora de la tarea inspectora, que a partir de entonces sería recurrente.

La LOGSE dedicaría su título IV a los factores que favorecen la calidad y mejora de la enseñanza, uno de los cuales sería la evaluación del sistema educativo, objeto del artículo 62. En él se establecía que la evaluación se aplicaría sobre los alumnos, el profesorado, los centros, los procesos educativos y la propia administración, y se creaba el Instituto Nacional de 
Calidad y Evaluación (INCE), aunque no se extendió mucho más en el tema que nos ocupa.

En el año 1994 el Ministerio de Educación y Ciencia publicaba el documento Centros Educativos y Calidad de la Enseñanza, preparatorio de la Ley Orgánica de la Participación, la Evaluación y el Gobierno de los Centros Docentes (LOPEG) de 1995, que planteaba un conjunto de 77 medidas para la mejora del sistema educativo, varias de las cuales incidían en el ámbito de la evaluación (MEC, 1994). El documento, que suponía un avance sobre los anteriores, analizaba el desarrollo de la evaluación educativa antes y después de la LOGSE, a la que consideraba (con justicia) un punto de inflexión. Además, insistía en considerar a los centros educativos objeto prioritario de atención, puesto que es en ellos donde se dilucida en última instancia la mejora de la calidad. En consecuencia, concedía una gran importancia a su evaluación, aun reconociendo que había otros ámbitos que requerían atención, como el profesorado, la función directiva, los resultados de los estudiantes, el proceso de reforma educativa y la propia administración. Como fruto de este análisis, proponía dos medidas relativas a la evaluación de los centros educativos: la generalización de la evaluación externa de los centros por medio del Plan EVA, al que se hará referencia más adelante (aun reiterando la necesidad de favorecer la autoevaluación de los propios centros); y la colaboración de los equipos directivos en los planes de evaluación de centros.

La LOPEG dedicó su artículo 29 a la evaluación de los centros docentes, encomendando a las administraciones educativas la elaboración y puesta en marcha de planes de evaluación que habrían de ser aplicados periódicamente a los centros sostenidos con fondos públicos, en los que participarían los equipos directivos y los distintos sectores de la comunidad educativa, y se llevarían a cabo principalmente a través de la inspección. Dichos planes deberían tomar en consideración los contextos socioeconómicos de los centros y sus recursos, adoptando una mirada amplia sobre las distintas dimensiones de su organización, funcionamiento y resultados. El consejo escolar de cada centro recibiría el informe de su evaluación, para que pudiese diseñar, con la colaboración de la administración, las actuaciones necesarias para resolver los problemas encontrados. Junto a ello, los centros habrían de evaluar anualmente su funcionamiento, de acuerdo con lo dispuesto por sus respectivas administraciones.

Unos meses más tarde, el 21 de febrero de 1996, se publicaba una orden ministerial sobre la evaluación de los centros docentes sostenidos con fondos públicos (BOE del 29 de febrero) que desarrollaba más extensamente lo dispuesto en la LOPEG sobre este asunto. En ella se establecía un mecanismo que combinaba la evaluación interna con la externa de los 
centros educativos. Así, al final de cada curso escolar, los centros debían llevar a cabo una autoevaluación que valorase la planificación y el desarrollo de su proyecto educativo, los proyectos curriculares de cada una de las etapas y ciclos impartidos, la programación general anual, las actividades complementarias y extraescolares, el proceso de enseñanza y la evolución del rendimiento escolar de los alumnos. Esta evaluación sería llevada a cabo por el consejo escolar (en los aspectos generales) y el claustro de profesores (en los aspectos relativos a la enseñanza y el aprendizaje). De manera complementaria, el centro sería evaluado cada cuatro o cinco años por los servicios de inspección, contemplándose la incorporación al equipo evaluador de algún director de otro centro, lo que abría la puerta a la evaluación por pares. La evaluación externa debería tomar en consideración el contexto socioeconómico y los recursos utilizados, y habría de llevarse a cabo sobre los procesos educativos, los resultados obtenidos, la organización, gestión y funcionamiento, y el conjunto de las actividades de enseñanza y aprendizaje. El informe de dicha evaluación debería presentarse al consejo escolar del centro y serviría de base para la elaboración de un plan de mejora.

En conjunto, como puede apreciarse, entre 1987 y 1996 el Ministerio de Educación y Ciencia fue construyendo un modelo coherente de evaluación de centros, cuyos rasgos más característicos fueron los siguientes: combinación de la evaluación interna con la externa; atribución de esta última a los servicios de inspección educativa; visión global, con el propósito de abarcar los diferentes aspectos y dimensiones de la vida de los centros; concepción democrática, incentivando la participación de los distintos sectores implicados; orientación hacia la mejora, mediante la elaboración de planes al efecto; fomento de la rendición de cuentas, aunque impidiendo la construcción de tablas de clasificación. La definición de este modelo fue posible gracias a la combinación de dos factores: la notable estabilidad de los equipos ministeriales que lo construyeron e impulsaron entre 1987 y 1996; y la amplitud de las competencias ministeriales, al no haberse completado todavía el proceso de transferencias en materia de educación (el denominado «territorio MEC», gestionado por el propio Ministerio, abarcaba prácticamente la mitad de la población escolar española). En otras circunstancias hubiese resultado mucho más complicado elaborar y aplicar un modelo semejante, como ocurriría en las etapas posteriores.

\section{El Plan EVA, paradigma de la evaluación de centros en los noventa}

El modelo que fue así construyéndose por iniciativa del Ministerio de Educación y Ciencia tuvo inmediata traducción práctica en la puesta en marcha del denominado Plan EVA. Dicho plan representó, sin duda, una muestra paradigmática de la actuación que el Ministerio se proponía 
llevar a cabo en lo que respecta a la evaluación de centros y constituyó una pieza central de su política en ese ámbito, por lo que merece una atención especial.

Con anterioridad a su diseño y puesta en marcha, en los años ochenta aparecieron en España algunos precedentes que contribuyeron a trazar las líneas generales del nuevo plan. Entre ellos cabe destacar el cuestionario denominado QUAFE-80, un instrumento de naturaleza académica creado en Cataluña para la evaluación formativa interna del funcionamiento de las escuelas de EGB, pero susceptible de ser utilizado por la inspección (López \& Darder, 1985). Y también puede mencionarse la iniciativa de evaluación externa puesta en marcha por el servicio de inspección de Asturias en el curso 1988-1989 y que se aplicó durante cuatro años, con una doble perspectiva de asesoramiento y diagnóstico, una finalidad eminentemente formativa y una concepción integral del centro (Pérez Collera, 1993). Ambos ejemplos comparten una característica destacable, consistente en la cooperación de actores procedentes del mundo académico y de la administración a la hora de diseñar planes e instrumentos para evaluar los centros docentes. Esa confluencia se apreciaría también en otras iniciativas posteriores.

El Plan EVA comenzó a concebirse tras la aprobación de la LOGSE y recibió el impulso que esa ley dio a las actividades de evaluación, siendo uno de sus primeros frutos en este campo. Su diseño comenzó a finales del año 1990, colaborando en el mismo un equipo de inspectores del Servicio de Inspección Central y otro de técnicos del Centro de Investigación, Documentación y Evaluación (CIDE), ambos del Ministerio de Educación y Ciencia. En el curso 1991-1992 se puso en marcha con carácter piloto en cuarenta centros públicos $\mathrm{y}$, tras valorar positivamente su primera aplicación y realizar algunas ligeras modificaciones, continuó aplicándose sin grandes cambios durante los cuatro cursos siguientes.

El plan, que ha sido presentado con detalle por dos de sus autores (Luján Castro \& Puente Azcutia, 1996), tenía carácter formativo, esto es, estaba dirigido fundamentalmente a la elaboración de un proyecto de mejora de los centros evaluados, más que a la rendición de cuentas, si bien proporcionaba información a la administración educativa acerca de la realidad de los centros escolares. Aunque pretendiese servir de estímulo para la puesta en marcha de procesos de autoevaluación, se trataba en realidad de una evaluación externa, llevada a cabo por un equipo de tres inspectores, uno de los cuales debía ser el responsable del seguimiento habitual del centro. De acuerdo con las pautas de la política antes analizada, pretendía tener un carácter democrático, implicando en su desarrollo a los diferentes sectores de la comunidad escolar y a todos los órganos de gobierno y representación del centro (equipo de dirección, consejo escolar, 
claustro docente, comisión de coordinación). Además, arrojaba una mirada global sobre el centro, abarcando los elementos contextuales y personales, los proyectos educativo y curricular, la programación anual, el modo de organización y funcionamiento, los procesos didácticos desarrollados en el aula y el centro y los resultados conseguidos.

Para llevar a cabo tal tarea, el equipo evaluador seguía un detallado protocolo de actuación y recurría a métodos tanto cualitativos como cuantitativos, aplicando una diversidad de técnicas e instrumentos, que se pueden consultar en la obra mencionada de Luján y Puente, la mayor parte de los cuales fueron construidos expresamente con esta finalidad. Desde el punto de vista técnico, se trataba de un plan cuidado, riguroso y completo, al que algunos criticaron su prolijidad y su quizás excesiva formalización. Pero tratándose de una experiencia pionera que pretendía generalizarse, hay que valorar positivamente el esfuerzo realizado para sistematizarlo y lograr su credibilidad y aceptación.

La evaluación culminaba cuando, tras llevar a cabo un conjunto pautado de visitas, reuniones y estudio de la información recopilada, el equipo evaluador presentaba su informe ante el consejo escolar del centro, que lo recibía y discutía. El informe debía contener propuestas de mejora, en cuya puesta en marcha debían comprometerse tanto el centro como la administración educativa. El plan de mejora resultante era una de las piezas clave de la evaluación.

A lo largo de sus cinco años de vida, el Plan EVA se aplicó en más de mil centros educativos del denominado «territorio MEC», compuesto entonces por las diez comunidades autónomas a las que aún no se habían transferido las competencias en materia de educación. Además, algunas de las otras siete (como fue el caso de Galicia, Andalucía o el País Vasco) aplicaron el mismo plan o variantes suyas, o se inspiraron en él para desarrollar sus propias actuaciones. En conjunto, puede afirmarse que constituyó la aplicación más representativa de la política común de evaluación de centros construida a comienzos de la década de los noventa e incluso dejó sentir su influencia en momentos posteriores.

\section{LA COMBINACIÓN DE LA EVALUACIÓN EXTERNA E INTERNA}

Como puede apreciarse, aunque la política diseñada entre 1987 y 1996 para la evaluación de centros en España había establecido como uno de sus principios clave la combinación de la perspectiva externa y la interna, la iniciativa más emblemática desarrollada a su amparo, el Plan EVA, lo fue en realidad de evaluación externa. Aunque esa decisión se justificase basándose 
en la necesidad de cultivar una casi inexistente cultura de la evaluación y de forzar a los centros docentes a abordar su autoevaluación como efecto de la evaluación externa, no puede dejar de subrayarse tal contradicción.

En todo caso, conviene señalar que la tensión existente entre la evaluación externa e interna de los centros educativos era claramente apreciable durante la década de los noventa en el panorama internacional. Los dos informes internacionales más destacados que recogían y analizaban el desarrollo experimentado por la evaluación de centros educativos en diversos países durante esos años (OECD, 1995; Nevo, 2002) llegaban a conclusiones similares: las escuelas que abordaban su propia evaluación de manera sistemática eran aún muy escasas, sin llegar a compensar la práctica predominante de evaluación externa que por entonces se extendía. Y Nevo llegaba a afirmar que «todos parecen odiar la evaluación externa mientras que nadie confía en la evaluación interna» (Nevo, 2002, p. 14).

$\mathrm{Y}$ es que, en efecto, el debate académico sobre ambos enfoques se había estimulado en esos años. Entre los académicos que habían defendido y promovido el uso de la autoevaluación como el instrumento más adecuado para mejorar los centros educativos, destacan el escocés John MacBeath (1999) y el israelí David Nevo, antes citado. Los dos ejercieron además un impacto apreciable sobre el debate que tenía lugar en España, debido a su participación en proyectos internacionales de los que nuestro país formaba parte y a su trabajo con colegas españoles. MacBeath animó una de las redes del proyecto de indicadores internacionales de la educación (INES) de la OECD, mientras que Nevo ocupó una posición relevante en la International Association for the Evaluation of Educational Achievement (IEA) y fue el editor jefe de la revista Studies in Educational Evaluation. Alguna de las obras más influyentes de Nevo, cuyas tesis a favor del diálogo entre la evaluación interna y la externa tuvieron notable presencia en el panorama internacional, llegó incluso a ser traducida al español, demostrando así su influencia (Nevo, 1997).

Otro planteamiento académico que encontró eco en España fue el de la evaluación democrática de las escuelas. Una de las autoras que más influyó en ese sentido fue Helen Simons, alguna de cuyas obras también fue traducida (Simons, 1999). El mismo principio defendieron otros autores que también trabajaron en España y difundieron sus ideas entre nosotros, como es el caso de Barry MacDonald (1995).

Los planteamientos de la evaluación democrática y del diálogo en la evaluación fueron retomados por universitarios españoles, como es el caso de Miguel Ángel Santos Guerra (1995). Y la evaluación de centros atrajo la atención de muchos investigadores, como pusieron de relieve algunos 
congresos que encontraron amplio eco en esos años (Sáez Brezmes, 1995; Villa Sánchez, 1996).

En todo caso, pese al interés teórico suscitado por la evaluación interna y la autoevaluación de los centros docentes, la práctica española se orientó mayoritariamente hacia la evaluación externa. Aunque la LOPEG y sus normas de desarrollo insistieron en la combinación de ambos enfoques, de las tres formas de combinación que Trond Alvik distinguía y que denominaba respectivamente paralela, secuencial y cooperativa (citado por MacBeath, 1999, p. 90), la política diseñada en los noventa optó por la secuencial, aunque sin asegurar los mecanismos que la hiciesen posible, con lo que muchas veces la práctica se quedó en el nivel de lo paralelo. Apenas en ningún caso se llegó al estadio de lo cooperativo, que hubiese sido el deseable y el más desarrollado.

\section{LA DESAPARICIÓN DE UNA POLÍTICA COMÚN Y LA DIVERSIFICACIÓN DE LAS PRÁCTICAS A PARTIR DE 1996}

El cambio de gobierno de 1996 tuvo repercusión directa sobre el desarrollo de la política de evaluación de centros educativos que se venía aplicando, por un conjunto de razones diversas. En primer lugar, aunque la LOPEG continuó vigente algunos años más (su derogación casi completa se produjo en 2002 por la Ley Orgánica de Calidad de la Educación [LOCE] y en lo que aún permaneció vigente por la Ley Orgánica de Educación [LOE] en 2006), su aplicación era aún incipiente, por lo que el nuevo gobierno no tuvo muchas dificultades para ralentizarla o incluso revertirla. En segundo lugar, se suspendió la aplicación del Plan EVA, su componente más emblemático, pese a la valoración positiva que había recibido por parte de la administración educativa y de los centros evaluados. En tercer lugar, la tarea prioritaria del rebautizado Ministerio de Educación y Cultura consistió hasta el año 2000 en finalizar el proceso de transferencia de competencias a las comunidades autónomas que aún no las habían recibido, en cumplimiento de lo previsto en el marco constitucional, lo que supuso un cambio notable en el proceso de construcción y desarrollo de políticas estatales comunes en aspectos relacionados con la gestión cotidiana del sistema educativo.

En consecuencia, la desaparición de un plan tan ampliamente difundido y el abandono de la política común que se había ido construyendo durante la década anterior supusieron inevitablemente la diversificación de las orientaciones políticas y la dispersión de las prácticas. Así, a partir de 1996, y hasta la actualidad, no ha existido una política estatal común para llevar a cabo la evaluación de los centros docentes, sino que la iniciativa ha estado (o dejado de estar) en manos de las administraciones autonómicas, 
a las que legítimamente les corresponde. Y las prácticas concretas han sido muy variadas, como demuestran algunos ejemplos relevantes.

El Ministerio de Educación y Cultura optó en un primer momento por la implantación de un modelo de gestión de calidad en la educación, basado en el desarrollado por la European Foundation for Quality Management (EFQM), que había avanzado unos años antes su principal impulsor desde el Ministerio (López Rupérez, 1994). Para llevar a cabo dicha iniciativa, comenzó promoviendo la elaboración de planes de mejora en los centros docentes, alcanzando la participación de 263 de ellos y publicando una selección de las mejores prácticas desarrolladas (MEC, 1998). Posteriormente, se elaboraron y publicaron materiales para la aplicación del modelo EFQM (MEC, 1997; MECD 2001), que, combinando la autoevaluación con la evaluación externa, intentaba dinamizar los centros y promover su mejora (Martínez Mediano \& Riopérez Losada, 2005). No obstante, el impacto de la iniciativa fue limitado, pues solo fueron once los centros que la iniciaron en 1997-1998 y algo más de cien al siguiente, manteniéndose la participación en cifras modestas, seguramente por la distancia que mantenía el modelo con la cultura organizativa habitual en los centros docentes. El hecho de reducirse la competencia directa del MECD a los territorios de Ceuta y Melilla a partir del año 2000 disminuyó drásticamente la incidencia de sus iniciativas.

Entre los planes puestos en marcha por las comunidades autónomas, merece la pena citar a Andalucía, quien siguió la senda que había abierto la LOPEG. En 1995 se publicó y sometió a debate el Plan Andaluz de Evaluación Educativa, que abarcaba un amplio conjunto de iniciativas, entre ellas, algunas encaminadas a evaluar los centros docentes. $\mathrm{Al}$ amparo de dicho plan se puso en marcha un programa de evaluación externa de escuelas, que se aplicó durante los cursos siguientes y fue objeto de una interesante metaevaluación, lo que constituye una rareza en los planes desarrollados durante estos treinta años, por lo que merece la pena ser destacado (Santos Guerra, 2008). Aunque estaba previsto combinar esta evaluación externa a cargo de la inspección con autoevaluaciones de los propios centros, esta previsión apenas se cumplió, como vemos que sucedió de modo bastante generalizado en otras iniciativas similares.

Por su parte, Canarias optó por un modelo original en España, basado en la práctica que algunos autores han denominado del «amigo crítico»o del «diálogo para la mejora» (Nevo, 1997). Tras alguna experiencia anterior, de signo más convencional, de carácter externo y metodología cuantitativa, el Instituto Canario de Evaluación y Calidad Educativa (ICEC), creado en 1995, puso en marcha en 1996 un Proyecto de evaluación interna y desarrollo de centros (EIDEC). Esta iniciativa introdujo varias novedades en la práctica habitual de la evaluación de centros: puso el énfasis en la 
autoevaluación, por encima de la evaluación externa; incorporó a expertos externos en los equipos de evaluación, aunque, a diferencia de otros planes, no procedían de la inspección, sino de los servicios de apoyo a los centros; puso en marcha un plan de formación de los equipos de autoevaluación y preparó documentos para apoyarles. Posteriormente, el EIDEC dio paso al Plan de Evaluación de Centros Docentes de Canarias, que comenzó a aplicarse en el curso 2000-2001 y tuvo una cobertura de unos cien centros al año, siendo también objeto de una metaevaluación que quedó recogida en documentos internos del ICEC.

Otras comunidades autónomas desarrollaron sus propios planes de evaluación de centros educativos, generalmente sin darles excesiva continuidad. Varias de ellas se inspiraron en el Plan EVA, promovieron especialmente la evaluación externa y concedieron un lugar destacado en ella a los servicios de inspección, contribuyendo a que las tareas de evaluación no se distinguieran claramente de las de supervisión y control.

Entre las iniciativas puestas en marcha en esos años, merece la pena destacar una de ellas promovida por una entidad privada y cuya larga vida se ha desarrollado fuera de las administraciones educativas, aunque manteniendo buenas relaciones con muchas de ellas. Se trata del Proyecto REDES, posteriormente articulado en el Instituto de Evaluación y Asesoramiento Educativo (IDEA) de la Fundación SM, que viene funcionando ininterrumpidamente desde 1997, habiendo extendido además su actuación a varios países iberoamericanos. Su origen se encuentra en el trabajo de varios miembros del equipo ministerial que diseñó la LOPEG, por lo que las líneas programáticas fundamentales son bastante similares a las que aquella ley establecía para la evaluación de centros, aunque lógicamente el modelo haya ido evolucionando con los años. El plan presenta varios rasgos distintivos: se basa en el funcionamiento en red de las escuelas participantes, lo que permite una comparación entre escuelas, aunque manteniendo la confidencialidad de los resultados y los informes; combina la aplicación de pruebas de rendimiento con análisis cualitativos y la elaboración de planes de mejora; y utiliza el concepto de "valor añadido» a la hora de valorar los resultados obtenidos por los centros (Marchesi \& Martín, 2002). Se trata de una experiencia de indudable interés y que ha venido desarrollándose durante veinte años, siendo la más longeva entre las existentes.

\section{EL RECURSO CRECIENTE A LOS INDICADORES DE RENDIMIENTO PARA EVALUAR LOS CENTROS}

Como puede apreciarse, la diversidad se convirtió en norma a partir de 1996 y la política de evaluación de centros docentes, que se había comenzado 
a construir de manera bastante homogénea, se fragmentó y evolucionó en múltiples direcciones. Desde el punto de vista político, las referencias de las nuevas leyes estatales de educación a esa realidad fueron disminuyendo paulatinamente. La LOCE reprodujo en su artículo 101 casi textualmente el artículo 29 de la LOPEG acerca de la evaluación de centros, aunque remitiendo su desarrollo a las diversas administraciones educativas, como no podía ser de otro modo tras el traspaso de las competencias en materia de educación. Posteriormente, la LOE redujo considerablemente esa referencia en su artículo 145, estableciendo solamente que las administraciones educativas podrían [sic] elaborar y realizar planes de evaluación de centros, disposición que fue mantenida por la Ley Orgánica para la Mejora de la Calidad Educativa (LOMCE) de 2013. Aunque esa reducción de referencias tuviese sentido tras el proceso de descentralización registrado, el Ministerio demostraba haber perdido la iniciativa que un día pudo tener en ese ámbito, sin que todas las comunidades autónomas la ejercitasen en la práctica. No obstante, hay que subrayar que algunas de ellas incorporaron diversas previsiones acerca de la evaluación de centros en las nuevas leyes autonómicas de educación que vieron la luz en la primera década del siglo, poniéndola en conexión con el fomento de la autonomía de los centros y la rendición de cuentas. Aunque sea de pasada, no se puede dejar de señalar que las limitaciones de nuestras políticas de evaluación de centros guardan también relación con insuficiencias tales como el limitado desarrollo de la autonomía de nuestros centros docentes, muchas veces proclamada, pero poco promovida en la práctica, o la carencia de unas políticas más coherentes de profesorado que permitan conformar una renovada profesión docente.

En cualquier caso, esas tres leyes estatales introdujeron una novedad relevante que acabó influyendo sobre las políticas (ahora autonómicas) de evaluación de centros docentes. Se trata de las nuevas evaluaciones generales de diagnóstico que comenzaron a realizarse en las etapas de Educación Primaria y Educación Secundaria Obligatoria (ESO). Con ligeras variaciones en su concepción, las tres leyes promovieron de hecho la aplicación de pruebas de rendimiento a los estudiantes de ciertos cursos de las etapas mencionadas, si bien es cierto que con algunas diferencias reseñables: mientras que la LOE dispuso que en ningún caso los resultados de estas evaluaciones podrían utilizarse para establecer clasificaciones de centros, la LOMCE sustituyó las evaluaciones generales de diagnóstico por las denominadas evaluaciones individualizadas y dispuso la publicación de los resultados de los centros docentes según indicadores educativos comunes para todos ellos.

Las comunidades autónomas comenzaron a diseñar este tipo de pruebas y a aplicarlas. Además, algunas de ellas utilizaron sus resultados 
para proporcionar información sobre los centros, impulsando en ocasiones la comparación explícita o implícita entre ellos. El caso más señalado es quizás el de la Comunidad de Madrid, que puso en marcha en 2005 las pruebas de Conocimientos y Destrezas Indispensables (CDI) en $6 .^{\circ}$ curso de Educación Primaria y 3..$^{\circ}$ de ESO, cuya última aplicación se realizó en 2015. Las pruebas CDI sirvieron para proporcionar información a las autoridades educativas y a los centros acerca de los resultados obtenidos. Al abarcar a toda la población de los cursos señalados, proporcionaron además datos individualizados para cada alumno, que se incluyeron en sus expedientes. Los resultados de los centros debían servir de base para la elaboración de planes de mejora, que habrían de incorporarse a la programación anual, aunque lo cierto es que el proceso no se sistematizó, por lo que se ha ido llevando a cabo de modos muy diversos. A partir de 2011, la Comunidad de Madrid comenzó a publicar en su web los resultados obtenidos por los centros en dichas pruebas, lo que permitió la elaboración de clasificaciones por parte de los medios de comunicación y provocó intensos debates.

Con planteamientos algo diferentes, también la Comunidad de Andalucía ha desarrollado diversas pruebas de rendimiento: las de diagnóstico, adaptadas a la LOE; las de final de etapa, adaptadas a la LOMCE; o las pruebas ESCALA, dirigidas al alumnado de $2 .^{\circ}$ de Primaria. A partir de los resultados de dichas pruebas, la Agencia Andaluza de Evaluación Educativa (AGAEVE) elabora unos informes de centro, que deben ser analizados y trabajados por estos con vistas a adoptar medidas para su mejora, y que en ocasiones van acompañados de informes para las familias.

Por su parte, la Generalitat de Cataluña ha puesto en marcha el plan de Avaluació Anual de Centres (AVAC), desarrollado por la inspección, de carácter censal y basado en el cálculo de un sistema de indicadores del centro (SCI) que abarca varias de sus dimensiones. Los centros reciben un informe anual que incluye los datos que obtienen en dichos indicadores y un conjunto de propuestas, lo que les permite reflexionar sobre su situación y adoptar medidas de mejora. Este plan convive con la Avaluació Global Diagnóstica (AGD), que se aplica solo a algunos centros seleccionados por sus características especiales.

Y en esa misma línea se orienta la reciente difusión del proyecto PISA para Centros Educativos (Sanz Labrador \& Gil Escudero, 2014) en diversas comunidades autónomas, de cuyo impacto real aún no se puede realizar una valoración ajustada.

El impacto de pruebas e indicadores de rendimiento sobre las políticas de evaluación de centros resulta indudable. De hecho, en 
algunas comunidades autónomas se han convertido en el instrumento fundamental para evaluar a los centros docentes. El rendimiento que logran los estudiantes se convierte en pieza angular del análisis, y los informes de resultados, mayoritariamente cuantitativos, aunque a veces acompañados de otros datos de contexto e indicadores de procesos, se consideran el elemento central sobre el que deben reflexionar los centros. La responsabilidad de estas evaluaciones suele recaer en la inspección, con algunas excepciones, como la AGAEVE en Andalucía. La autoevaluación se presume como efecto automático de la evaluación externa, pero no suele recibir atención específica ni apoyarse con instrumentos preparados al efecto. Y, además, la elaboración de planes de mejora se convierte muchas veces en un proceso formal que recibe menos atención de la debida. Ese es el modelo preeminente en la actualidad, bastante alejado del proyectado hace treinta años, como se puede apreciar.

\section{CONCLUSIONES}

En los últimos treinta años se ha manifestado, tanto en España como internacionalmente, un interés genuino por la evaluación de los centros docentes, considerándola un instrumento esencial para mejorar la calidad de la educación. Pero el recorrido expuesto en estas páginas autoriza a decir que, al menos en España, se ha tratado de un interés más teórico que real, puesto que apenas ha ido acompañado de la definición y puesta en práctica de políticas educativas con esa finalidad.

En los años noventa se comenzó a construir una política de evaluación de centros que, a pesar de sus limitaciones, diseñó un modelo común para llevarla a cabo en la mayor parte del territorio español. Si bien es cierto que su aplicación puso más énfasis en la dimensión externa que en la autoevaluación y que su pretendido carácter democrático se limitó a la puesta en marcha de mecanismos de consulta y discusión de informes, el modelo comenzaba a dar sus frutos cuando se interrumpió, debido al cambio de gobierno y a la finalización del proceso de transferencias de competencias educativas a las comunidades autónomas, de acuerdo con lo previsto en el marco constitucional. La consecuencia de ese giro fue que la política bastante homogénea que se estaba construyendo se diversificó en múltiples direcciones, siguiendo los desarrollos legítimamente impulsados por las distintas comunidades autónomas.

En los años que llevamos transcurridos del siglo XXI, las políticas de evaluación de centros tienen lógicamente carácter autonómico, aunque sean pocas las comunidades que han adoptado políticas propias al respecto. La evaluación de centros ha venido marcada sobre todo por la tendencia 
cada vez más extendida a poner el foco de atención en los resultados obtenidos por los estudiantes en pruebas estandarizadas de evaluación y en el rendimiento logrado por los centros. A lo sumo, se han incorporado cálculos del valor añadido por los centros o se han completado los datos centrales cuantitativos con algunos otros cualitativos. Pero lo más llamativo es que, aun considerando que el objetivo de dichas políticas debe consistir en reflexionar y aprender para promover la mejora, se ha concedido poca importancia a esa tarea en la práctica, pues se ha incorporado la evaluación de centros a la función inspectora y se han descuidado los mecanismos de autoevaluación.

En todo caso, la valoración del impacto de dichas políticas de evaluación de centros está aún por hacer. La diversidad de iniciativas y experiencias constituye una indudable riqueza, pero no sabemos cómo pueden estar repercutiendo sobre la mejora del funcionamiento de los centros ni de la enseñanza que imparten. Es esta una línea todavía pendiente de investigación, tan interesante como valiosa, que nos permitirá conocer mejor el potencial y los efectos de la práctica de la evaluación de los centros educativos.

En resumen, la política de evaluación desarrollada en estos treinta años en España se ha caracterizado por los avances y retrocesos, los cambios de orientación, la falta de coherencia entre las intenciones y la práctica y la diversidad de las acciones desarrolladas. El reconocimiento de la existencia de experiencias valiosas no elimina esta sensación general de estar ante un desarrollo insuficiente. Continuamos escuchando alegatos en favor de la importancia de evaluar a los centros, pero no se aprecia la adopción de políticas coherentes en esa dirección ni una evaluación rigurosa de su impacto. 


\section{REFERENCIAS BIBLIOGRÁFICAS}

De la Orden, A. (1988). La calidad de la educación. Bordón, 40 (2), 149-161.

House, E.R. (1993). Professional Evaluation. Social Impact and Political Consequences. Newbury Park, London \& New Delhi: SAGE.

López, J.A. y Darder, P. (1985). QUAFE80. Cuestionario para el análisis del funcionamiento de la escuela. Barcelona: Onda.

López Rupérez, F. (1994). La gestión de calidad en educación. Madrid: La Muralla.

Luján Castro, J. y Puente Azcutia, J. (1996). Evaluación de Centros Docentes. El Plan EVA. Madrid: Ministerio de Educación y Ciencia.

MacBeath, J. (1999). Schools Must Speak for Themselves. The case for school self-evaluation. London \& New York: Routledge.

MacDonald, B. (1995). La evaluación como servicio público: perspectivas de futuro. En M.J. Sáez Brezmes (Ed.). Conceptualizando la educación en España (pp. 15-23). Alcalá de Henares: Universidad de Alcalá-ICE.

Marchesi, A. y Martín, E. (1998). Calidad de la enseñanza en tiempos de cambio. Madrid: Alianza Editorial.

Marchesi, A. y Martín, E. (Comp.) (2002). Evaluación de la educación secundaria. Fotografía de una etapa polémica. Instituto IDEA. Madrid: Fundación SM.

Martínez Mediano, C. \& Riopérez Losada, N. (2005). El modelo de excelencia en la EFQM y su aplicación para la mejora de la calidad de los centros educativos. Educación XXI, 8, 35-65.
Ministerio de Educación y Ciencia (1969). La educación en España. Bases para una política educativa. Madrid: MEC.

Ministerio de Educación y Ciencia (1987). Proyecto para la reforma de la enseñanza. Propuesta para debate. Madrid: MEC.

Ministerio de Educación y Ciencia (1989). Libro Blanco para la Reforma del Sistema Educativo. Madrid: MEC.

Ministerio de Educación y Ciencia (1994). Centros Educativos y Calidad de la Enseñanza. Propuesta de actuación. Madrid: MEC.

Ministerio de Educación y Cultura (1997). Modelo Europeo de Gestión de Calidad. Madrid: MEC-Argentaria.

Ministerio de Educación y Cultura (1998). Planes anuales de mejora. Prácticas de Buena Gestión en Centros Educativos Públicos. Madrid: MEC.

Ministerio de Educación, Cultura y Deporte (2001). Modelo Europeo de Excelencia. Adaptación a los centros educativos del modelo de la Fundación Europea para la Gestión de Calidad. Madrid: MECD.

Nevo, D. (1997). Evaluación basada en el centro. Un diálogo para la mejora educativa. Bilbao: Mensajero.

Nevo, D. (Ed.) (2002). Schoolbased evaluation: Evaluation in an international perspective. Amsterdam, Boston, London \& al.: JAI-Elsevier (Advances in Program Evaluation, vol. 8).

Organisation for Economic Co-operation and Development (1992). High-Quality Education and Training for All. Paris: OECD. 
Organisation for Economic Co-operation and Development (1994). Quality in Teaching. Paris: OECD-Centre for Educational Research and Innovation.

Organisation for Economic Co-operation and Development (1995). Schools under Scrutiny. Paris: OECD-Centre for Educational Research and Innovation.

Pérez Collera, A. (1993). El Plan de evaluación de centros del S.I.T.E. de Asturias. Una propuesta heterodoxa sin pretensiones. Bordón, 45 (3), 321-330.

Sáez Brezmes, M.J. (Ed.). (1995). Conceptualizando la educación en España. Alcalá de Henares: Universidad de Alcalá-ICE.

Santos Guerra, M.A. (1995). La evaluación: un proceso de diálogo, evaluación y mejora. Archidona, Málaga: Aljibe.

Santos Guerra, M.A. (Coord.) (2003). Trampas en educación. El discurso sobre la calidad. Madrid: La Muralla.
Santos Guerra, M.A. (Coord.) (2008). Nieve y barro. Metaevaluación del Plan de Evaluación de Centros Escolares de Andalucía. Alcalá de Guadaira, Sevilla: Editorial MAD.

Sanz Labrador, I. y Gil Escudero, G. (2014). PISA para Centros Educativos: Un instrumento para el fomento del éxito escolar de los centros. Participación Educativa, 3 (4), 29-37.

Simons, H. (1999). Evaluación democrática de instituciones escolares. Madrid: Morata.

Varios autores (1994). Calidad de la Educación. Revista Iberoamericana de la Educación, 5, 7-157.

Villa Sánchez, A. (Coord.) (1996). Dirección participativa y evaluación de centros. II Congreso Internacional sobre Dirección de Centros Docentes. Bilbao: Mensajero, ICE Universidad de Deusto. 


\section{PERFIL ACADÉMICO Y PROFESIONAL DEL AUTOR}

Alejandro Tiana-Ferrer. Doctor en Filosofía y Letras (Pedagogía) por la Universidad Complutense, catedrático y actual rector de la UNED. Viene trabajando desde 1980 en las áreas de Historia de los Sistemas Educativos Contemporáneos, Política y Legislación Educativa, Educación Comparada y Evaluación de la Educación. Ha desempeñado varias responsabilidades en la UNED, además de diversos cargos en el Ministerio de Educación, entre ellos Secretario General de Educación (2004-2008). Es autor o coautor de 23 libros y más de 200 artículos o capítulos de libros sobre diversos temas de sus especialidades.

Dirección del autor: Dpto. Historia de la Educación y Educación Comparada

Facultad de Educación. UNED

C/ Juan del Rosal, 14

28040 Madrid (España)

E-mail: atiana@edu.uned.es

Fecha Recepción del Artículo: 26. Febrero. 2018

Fecha Aceptación del Artículo: 23. Marzo. 2018

Fecha Revisión para publicación: 25. Marzo. 2018 